\title{
HIGHER-HARMONIC ADAPTATION AND THE DETECTION OF SQUAREWAVE GRATINGS*
}

\author{
Mark W. Greenlee and Svein Magnussent \\ Neurologische Klinik mit Abteilung für Neurophysiologie, Hansastr. 9 , \\ 7800 Freiburg im Breisgau, F.R.G.
}

(Received 28 November 1985; in revised form 29 May 1986)

\begin{abstract}
Adaptation to a high contrast sinewave grating of $1 \mathrm{c} / \mathrm{deg}$ spatial frequency causes a large increase in the contrast threshold for a $1 \mathrm{c} / \mathrm{deg}$ test grating, but fails to raise the threshold for a squarewave grating of $0.33 \mathrm{c} / \mathrm{deg}$, although the sensitivity of the "channel" tuned to both the third and fifth harmonic components of the squarewave test grating should be thoroughly suppressed. Following sequential adaptation to sinewave gratings of 1 and $3 \mathrm{c} / \mathrm{deg}$ spatial frequency, detection of squarewave gratings at $0.33 \mathrm{c} / \mathrm{deg}$ likewise remains unaffected. In contrast, after adaptation to a $0.33 \mathrm{c} /$ deg squarewave grating with missing fundamental the contrast threshold for a squarewave test grating of the same frequency is increased by $0.25 \mathrm{log}$ unit, although the higher harmonic component frequencies are less affected than by sequential sinewave adaptation. The results suggest that independent spatial frequency channels detecting harmonic components are not alone sufficient to account for the visibility of low frequency squarewaves.
\end{abstract}

Linear filtering Adaptation Low frequency detection Complex gratings

\section{INTRODUCTION}

At low spatial frequencies, sensitivity to sinewave gratings falls off linearly, but remains approximately constant for squarewave gratings (Campbell and Robson, 1968). These differences may be explained in terms of linear filtering theory, according to which the sensitivity to the higher harmonics $(3 f, 5 f$, etc.) rather than the fundamental frequency $(f)$ determines threshold for the low-frequency squarewave grating. Campbell and Robson (1968) further showed that a single-channel system, represented by a single contrast sensitivity function, could not account for the difference between the sensitivity to sinewave and squarewave gratings at low spatial frequencies. They, therefore, suggested the existence of multiple channels, each tuned to a different spatial frequency, acting independently (see also, Sachs et al., 1971; Graham and Nachmias, 1971). A complex grating is, accordingly, either detected by the most sensitive channel or as a result of probability summation over channels (Wilson and Bergen, 1979). For squarewave gratings of low spatial frequencies this means that the channels with

*Part of these findings were presented at the 8th European Conference on Visual Perception, Peniscola, Spain, September, 1985.

†Present address: Institute of Psychology, University of Oslo, Oslo 3, Norway. peak sensitivities at the third, fifth, and higher harmonic components might be employed to detect the squarewave grating at threshold (see Jaschinski-Kruza and Cavonius, 1984).

Further evidence of the multichannel model was reported by Pantle and Sekuler (1968) and Blakemore and Campbell (1969), who showed that after adapting to a suprathreshold grating, the contrast threshold was elevated for test gratings of the same or similar spatial frequencies. This adaptation effect exhibited a constant bandwidth for the range of adapting frequencies tested. A set of $n$ such channels of narrow to medium bandwidth and maximal sensitivity at prescribed spatial frequencies might encode the various frequency components of the retinal image. A fairly limited number of channels is usually assumed (Wilson and Bergen, 1979; Watson and Robson, 1981; Sekuler et al., 1984; Wilson and Gelb, 1984).

Campbell et al. (1981) more recently put forth the idea of a "watershed" in spatial vision. According to this idea, harmonic analysis is conducted for spatial frequencies above $1 \mathrm{c} / \mathrm{deg}$, whereas local, gradient detection is done below $1 \mathrm{c} /$ deg. Jaschinski-Kruza and Cavonius (1984) have demonstrated, however, that gradient detectors are not necessary to account for lowfrequency detection, since this can be accurately predicted by their space-domain model. It remains, therefore, to be shown whether a low- 
frequency squarewave is detected by independent channels tuned to its harmonic components.

\section{Rationale for the present experiment}

To test whether linear filtering theory can account for the visibility of low-frequency, complex (squarewave) gratings, we adapted our subjects to sinewave gratings whose spatial frequencies were equal to the higher harmonic frequencies of a squarewave test grating. The fundamental frequency of this grating was in the low-frequency region of the contrast sensitivity function where sensitivity to squarewaves exceeds that to sinewaves by more than $4 / \pi$ (cf. Campbell and Robson, 1968). Thus, at threshold the fundamental component of the squarewave remains below its detection threshold, so that only the higher harmonics contribute to detection. By choosing the adapting and test frequencies so that the "channel" tuned to the 3rd and 5th harmonics and the "channel" tuned to the 7-13th harmonics of the squarewave test grating (assuming a bandwidth of about 1.2 octave) were reduced in their sensitivity via adaptation, the contrast threshold for a low spatial frequency squarewave grating should approach that for a sinewave test grating of the same spatial frequency.

To adapt to this large range of higher harmonic frequencies, we introduce a technique of sequential adaptation. In a recent paper (Magnussen and Greenlee, 1986), we showed that brief rest pauses can be taken during adaptation without affecting the resultant elevation in threshold, provided that the total adaptation time is kept constant. These rest pauses can be utilized for adantation to another spatial fre- of visual angle. Viewing was binocular. A chin and forehead rest assurred constant viewing distance and head orientation.

Contrast thresholds were measured using a two interval forced-choice procedure. The grating was displayed for $1 \mathrm{sec}$ in one of the two $2.5 \mathrm{sec}$ intervals, which were delineated by auditory signals. The subject's task was to signal, by pressing one of two buttons. in which interval the grating was presented. Contrast thresholds were measured based on a microprocessor controlled staircase procedure in which contrast was decremented by $0.1 \mathrm{log}$ unit following a correct response and incremented by $0.3 \log$ unit following a wrong response. The contrast of the test gratings at the reversal points was averaged giving a mean threshold value for that run. This resulted in threshold values with a standard error that varied from 0.05 to $0.15 \log$ unit for unadapted and adapted thresholds.

Three experiments were conducted. In the first experiment, subjects adapted for $4 \mathrm{~min}$ to a stationary sinewave grating of 0.4 contrast having a spatial frequency of either 1 or $3 \mathrm{c} / \mathrm{deg}$. In the second experiment, subjects adapted for $8 \mathrm{~min}$ to these two sinewave gratings, presented sequentially, each spatial frequency for $2.5 \mathrm{sec}$, over the $8 \mathrm{~min}$ adaptation period. In a third experiment, subjects adapted for $4 \mathrm{~min}$ to a $0.33 \mathrm{c} / \mathrm{deg}$ squarewave grating with missing fundamental. This adapting grating contained all of the higher harmonics of the squarewave test grating with amplitudes inversely proportional to their order.

During adaptation, subjects moved their eyes along a $6 \mathrm{deg}$ fixation circle to avoid inducing retinal afterimages. After the adaptation period,

the screen returned to zero contrast and an having to present complex adapting gratings.

\section{METHOD}

Vertical sinewave gratings were produced on a CRT (Joyce Electronics) by modulating the luminance of the individual raster lines. This was done by multiplying the values of two digital-to-analog converters (computer interface; GRSYS2, Cambridge Research) controlling the voltage of the raster display. Mean luminance of the screen was $150 \mathrm{~cd} / \mathrm{m}^{2}$ and it was surrounded by a circular mask of $30 \mathrm{~cd} / \mathrm{m}^{2}$. The viewing distance was $57 \mathrm{~cm}$, at which the grating produced on the CRT subtended $22 \mathrm{deg}$ period, the subject readapted for $15 \mathrm{sec}$, where the grating (or gratings) used in the initial adaptation period was (were) again presented. Test and readapt periods were cycled 40 times yielding approximately 8-10 reversals. Preliminary testing indicated that these procedures induced a steady state of adaptation throughout the test-readapt series (lasting about $15 \mathrm{~min}$ ). Adequate time was given between runs to assure complete recovery from adaptation. The various test conditions were conducted in counterbalanced order to cancel possible sequence effects. Subjects were the first author M.W.G. and two naive subjects, who wore their spectacle corrections during the experiments. 


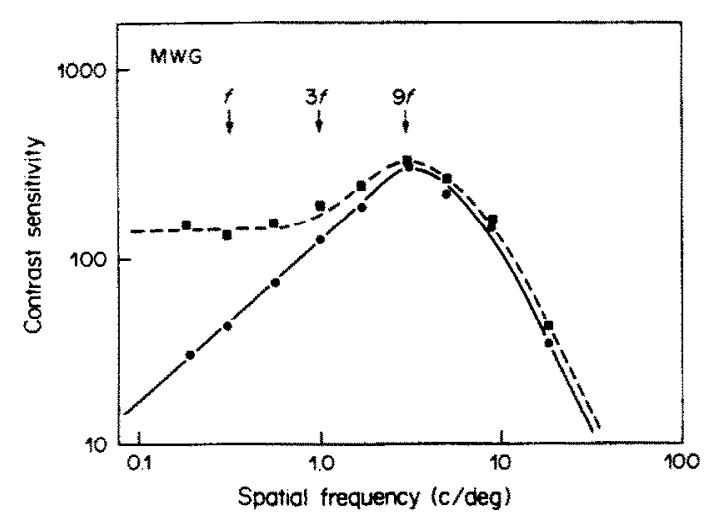

Fig. 1. Contrast thresholds for sinewave (circles) and squarewave (squares) gratings as a function of spatial frequency. Arrows denote position on the frequency axis of the fundamental $(f)$, third $(3 f)$ and ninth $(9 f)$ harmonic frequencies of the squarewave test grating used in the experiments depicted in Figs 3-5. Data from M.W.G.

\section{RESULTS}

Contrast sensitivity to sinewave and squarewave gratings

Figure 1 shows, for observer M.W.G., the contrast thresholds for sinewave (O) and squarewave ( $D$ ) gratings as a function of spatial frequency. For our conditions, the contrast sensitivity function peaks near $3 \mathrm{c} / \mathrm{deg}$ for both waveforms. For sinewave gratings, sensitivity is attenuated at lower spatial frequencies, whereas for squarewave gratings, sensitivity remains near the value for $1 \mathrm{c} / \mathrm{deg}$. At $0.33 \mathrm{c} / \mathrm{deg}$, the fundamental frequency of the critical test grating used in our experiments, there is a sensitivity difference of $0.5 \mathrm{log}$ unit, yielding a difference between waveforms of $0.4 \mathrm{log}$ unit after the $4 / \pi$ fundamental enhancement in amplitude of the squarewave grating has been subtracted (cf. Campbell and Robson, 1968). In agreement with original observations by Campbell and Robson (1968), sensitivity to squarewaves above $1 \mathrm{c} / \mathrm{deg}$ correspond to the enhancement expected by the $4 / \pi$ increase in their fundamental amplitudes.

Spatial-frequency selectivity of simple and sequential adaptation

To evaluate the method of sequential adaptation, we first compared contrast threshold elevations for sinewave test gratings between 0.33 and $9 \mathrm{c} / \mathrm{deg}$ following $4 \mathrm{~min}$ of adaptation to either a $1 \mathrm{c} / \mathrm{deg}(\square)$ or a $3 \mathrm{c} / \mathrm{deg}(O)$ sinewave adapting grating to that elicited by $8 \mathrm{~min}$ of sequential adaptation to 1 and $3 \mathrm{c} / \mathrm{deg}(\mathrm{O})$. The

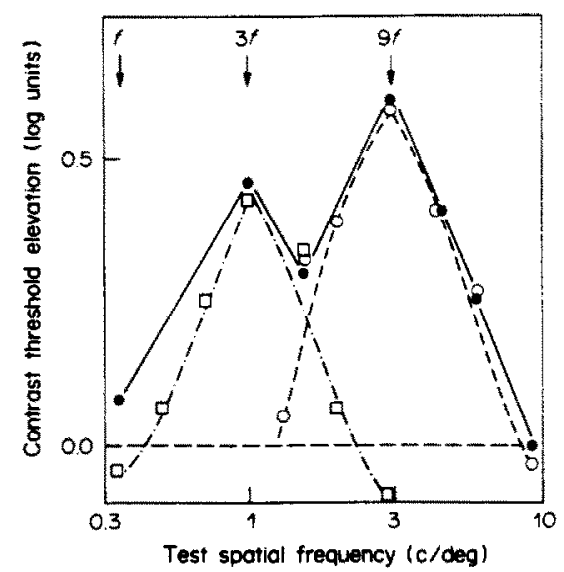

Fig. 2. Log threshold elevation as a function of test frequency is shown following $4 \mathrm{~min}$ adaptation at $3 f(\square)$ and $9 f(O)$. Also shown are elevations in threshold following $8 \mathrm{~min}$ of sequential adaptation at $3 f$ and $9 f(0)$. Data from M.W.G.

results are shown in Fig. 2 as open and solid symbols, respectively. For a particular test frequency, threshold elevation is defined as the difference between the log adapted and the $\log$ unadapted thresholds. The results indicate an adaptation effect having a bandwidth of 1.3 octaves at half-height for the channel centered at $1 \mathrm{c} / \mathrm{deg}$ and approximately 1.0 octave for the channel centered at $3 \mathrm{c} / \mathrm{deg}$. This compares with several previous reports (see Blakemore and Campbell, 1969; Björklund and Magnussen, 1981; Georgeson and Harris, 1984). The continuous lines show the effect of sequential adaptation to 1 and $3 \mathrm{c} / \mathrm{deg}$. This composite effect closely approximates the envelope of the elevation in threshold induced by $4 \mathrm{~min}$ of continuous adaptation to each 1 and $3 \mathrm{c} / \mathrm{deg}$ grating separately. Thus, sequential adaptation is just as effective in inducing an elevation in threshold as is continuous adaptation of equal exposure duration (cf. Magnussen and Greenlee, 1986). Thus sequential adaptation to 1 and $3 \mathrm{c} / \mathrm{deg}$ should reduce the sensitivity to the third through 13th harmonic frequencies of the $0.33 \mathrm{c} / \mathrm{deg}$ squarewave grating.

Effect of higher-harmonic adaptation on sensitivity to squarewave gratings

We first tested the effect of simple adaptation to $1.0 \mathrm{c} / \mathrm{deg}(3 f)$ and $3.0 \mathrm{c} / \mathrm{deg}(9 f$; corresponding to the peak of the contrast sensitivity function, see Fig. 1) on the contrast threshold for $0.33 \mathrm{c} / \mathrm{deg}(f)$ squarewave test grating. The results are shown in Fig. 3 as open columns. Also shown are control data for $0.33,1.0$ and 


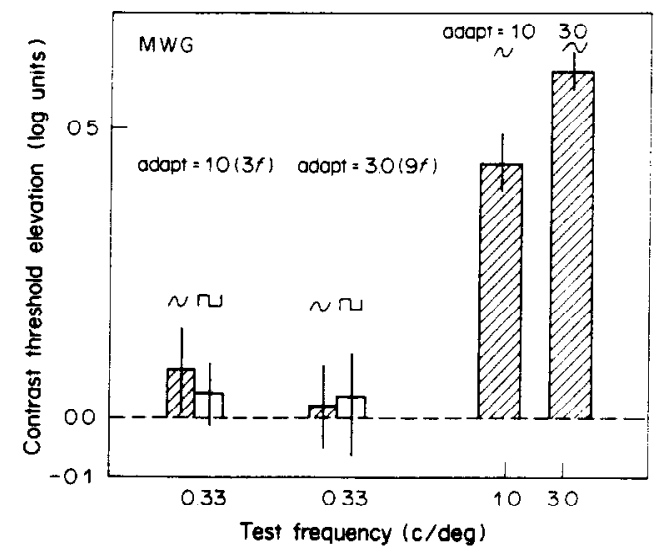

Fig. 3. Log threshold elevation following $4 \mathrm{~min}$ adaptation to sinewave grating of 1 or $3 \mathrm{c} / \mathrm{deg}$ is shown for various test gratings. Right: test grating $=1$ or $3 \mathrm{c} / \mathrm{deg}$ sinewave (i.e. identical to adapting frequency). Left: test grating $=0.33 \mathrm{c} / \mathrm{deg}$ sinewave (hatched) and squarewave (open columns) grating. Error bars depict $\pm 2 \mathrm{SE}$. Data from M.W.G., mean values of 5 runs.

$3 \mathrm{c} / \mathrm{deg}$ sinewave test gratings (hatched columns). Vertical lines indicate $\pm 2 \mathrm{SE}$ based on five threshold determinations resulting from five separate runs. Thus the variability of the mean threshold from each run, rather than the individual reversal points, are shown. Since $0.33 \mathrm{c} / \mathrm{deg}$ is outside of the bandwidth of adaptation to $1.0 \mathrm{c} / \mathrm{deg}$ (cf. Fig. 2), any adaptation effect observed on the $0.33 \mathrm{c} / \mathrm{deg}$ square wave grating would have to be attributed to decreased sensitivity to the higher harmonic frequencies. However, there is no significant adaptation effect on the $0.33 \mathrm{c} / \mathrm{deg}$ squarewave sensitivity (open columns). This lack of elevation in threshold for the squarewave test grating cannot be accounted for by a counteractive facilitation in sensitivity at the fundamental frequency (De Valois, 1977; Kelley and Burbeck, 1980), since there is no increase in sinewave sensitivity at this frequency (hatched column, left), nor can it be attributed to a lack of adaptation at 1.0 or $3.0 \mathrm{c} / \mathrm{deg}$ (hatched columns, right).

Figure 4 shows the results of $8 \mathrm{~min}$ of sequential adaptation to 1 and $3 \mathrm{c} / \mathrm{deg}$ (upper panel, subject M.W.G.; lower panel, subject D.B.). The columns on the right side show the threshold elevation at the test frequencies of 1 and $3 \mathrm{c} / \mathrm{deg}$, the columns on the left show the change in threshold for sinewave (hatched column) and squarewave (open column) test gratings at $0.33 \mathrm{c} / \mathrm{deg}$. Again, no change is observed in the threshold for a squarewave grating at the fundamental frequency $(0.33 \mathrm{c} / \mathrm{deg})$ following adaptation to its higher harmonic com-
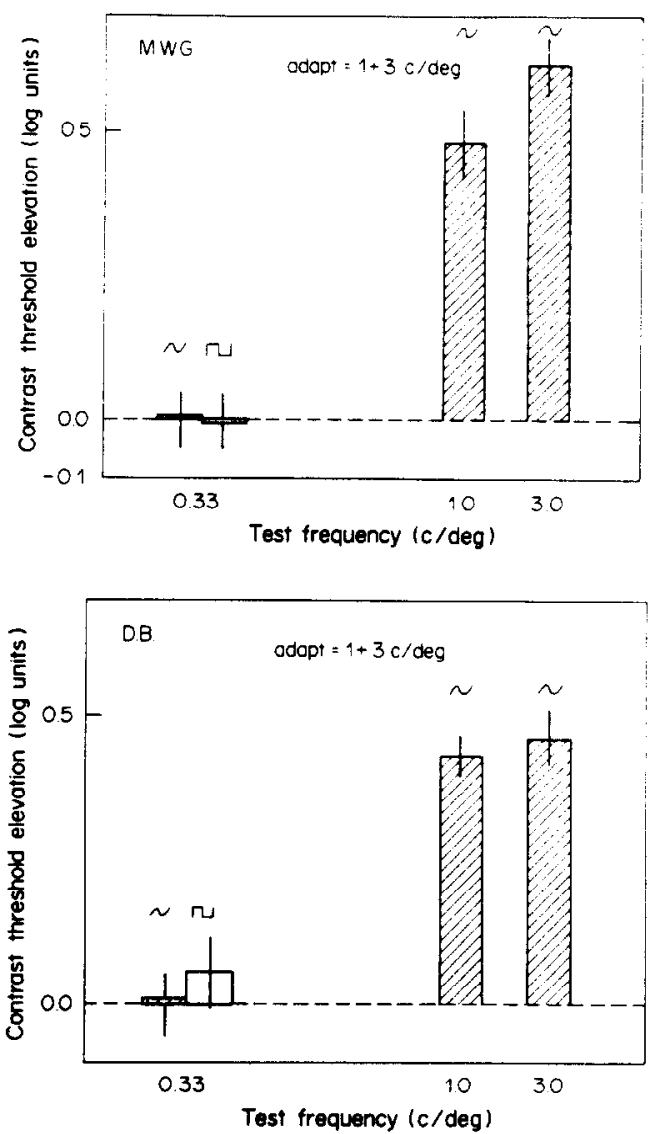

Fig. 4. Log threshold elevation following $8 \mathrm{~min}$ of sequential adaptation to sinewave gratings at 1 and $3 \mathrm{c} / \mathrm{deg}$ shown for various test gratings as in Fig. 3. Data from M.W.G. (upper panel) and D.B. (lower panel); mean values of 5 runs.

ponents. These findings demonstrate that adaptation at the harmonic frequencies of the squarewave test grating has no effect on the subsequent detection of that grating.

The results of the first two experiments suggest that "channels" sensitive to the spatial frequencies of the squarewave's harmonics are not responsible for the increased sensitivity to the squarewave's edges. To further explore the underlying mechanism(s) responsible for this sensitivity difference, we adapted our subjects to the edges of the squarewave grating given in a 0.33 squarewave with missing fundamental. Figure 5 compares this condition to the effect of sequential adaptation (taken from Fig. 4; subject M.W.G.). The squarewave with missingfundamental adapting grating causes less elevation in threshold for sinewave test gratings at $1 \mathrm{c} / \mathrm{deg}(3 f)$ and $3 \mathrm{c} / \mathrm{deg}$ (9f: open columns, right half) than sequential adaptation (hatched columns, right half). Nevertheless, the contrast threshold for squarewaves at $0.33 \mathrm{c} / \mathrm{deg}$ is increased by $0.25 \log$ unit. 


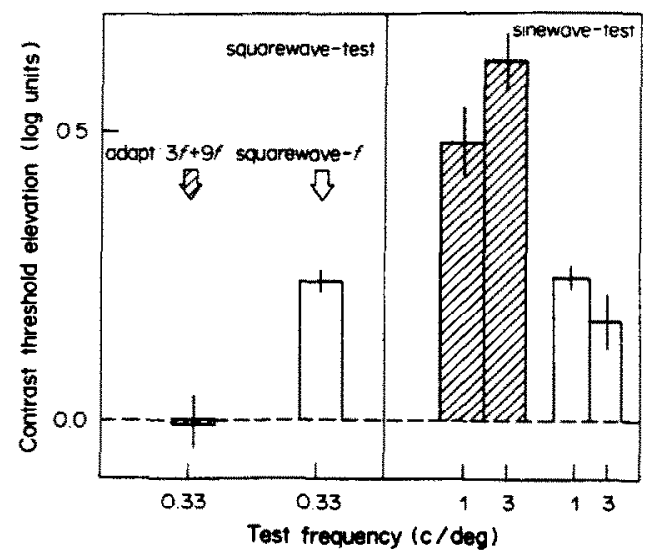

Fig. 5. Log threshold elevation following $4 \mathrm{~min}$ adaptation to a squarewave with missing fundamental (open columns) having a contrast of 0.4 . Hatched columns show data from Fig. 4 for comparison. Left half shows results for squarewave test gratings at $0.33 \mathrm{c} / \mathrm{deg}$. Right half shows threshold elevation at $1(3 f)$ and $3(9 f) \mathrm{c} / \mathrm{deg}$ component frequencies.

Data from M.W.G.; mean values of 5 runs.

\section{DISCUSSION}

Following $4 \mathrm{~min}$ adaptation to a highcontrast 1.0 or $3 \mathrm{c} / \mathrm{deg}$ sinewave grating and following $8 \mathrm{~min}$ of sequential adaptation to these two gratings, the thresholds for test gratings of the same spatial frequencies are increased by $0.4-0.6 \log$ unit, but no significant threshold change is observed for a $0.33 \mathrm{c} / \mathrm{deg}$ squarewave grating. According to the independent-channel hypothesis of spatial vision, initially proposed by Campbell and Robson (1968), squarewave gratings of low spatial frequencies are detected by channels independently sensitive to their higher harmonics. Accordingly, a decrease in sensitivity of about $0.2-0.4$ log unit would have been expected following harmonic adaptation (see Appendix for model predictions). Probability summation across channels and over space likewise predicts an elevation in detection threholds whenever the contribution of one of the active channels (e.g. that tuned to $3 f$ ) is suppressed (see Fig. 8 in appendix). It might be argued that suppressing the activity of a single channel does not suffice to elicit a significant change in the sensitivity to squarewave gratings, since presumably more than one channel is contributing to the detection of the squarewave. However, our sequential adaptation counters this objection since the harmonics $3 f-13 f$ are simultaneously affected. The results of these experiments show that after suppressing the sensitivity of the channels tuned to the squarewave's higher harmonics, the threshold for detecting a squarewave grating is unchanged. A revised version of the model presented by Jaschinski-Kruza and Cavonius (1984) predicted here a $0.4 \log$ unit decrease in sensitivity to the $0.33 \mathrm{c} / \mathrm{deg}$ squarewave (Fig. 8 in Appendix).

Adaptation to a squarewave with missing fundamental does, on the other hand, increase squarewave thresholds by $0.25 \mathrm{log}$ unit, although elevation at the component frequencies themselves (e.g. $3 f, 9 f$ ) was less than that elicited by sequential adaptation. Tolhurst (1972) and Nachmias et al. (1973) found that adaptation to squarewave gratings caused less effect at the component frequencies than that caused by sinewave adaptation to those frequencies, and Stromeyer and Klein (1974) and Klein and Stromeyer (1980) report that the decreased effect could be the result of inhibition between edge (asymmetric) and line (symmetric) mechanisms. These findings and those reported in the present paper seem to suggest that local aspects of the luminance profile (edges) are important in adaptation. Although sequential adaptation to sinewave gratings whose spatial frequencies are equivalent to the third and ninth harmonics of a squarewave test has no effect on the detection of that squarewave, adaptation to all higher harmonics presented simultaneously (i.e. the squarewave with missing fundamental, see Fig. 5) does have a significant effect on squarewave detection. Apparently, the adaptation caused by a stimulus in which the superposition of sinewave components results in an edge is not equivalent to the linear summation of adaptation effects caused by the individual harmonic components themselves. Therefore, our findings suggest that the visual system responds in a nonlinear way to the harmonic components of a complex pattern, depending on their relative spatial phases and, thus, on the luminance profile they form.

The present results further suggest that sequential adaptation might be a powerful technique to explore interactions between visual channels in the brain, without the confounding effects introduced by complex gratings (e.g. "beat" frequencies; Derrington and Badcock, 1986). We are presently using this technique to investigate the type and extent of interactions between channels in the spatial-frequency and orientation domains.

Acknowledgements - This research was supported by the Deutsche Forschungsgemeinschaft (SFB 325, B4) and by a research fellowship from the Alexander von Humboldt Stiftung (Bonn, F.R.G.) to S. M. We thank L. Spillmann 
for providing laboratory facilities and helpful comments. The authors would like to thank Sylvia Strauss and Doris Braun for serving as observers. The authors also thank W. Jaschinski-Kruza (Dortmund) for performing the calculations for the model predictions.

\section{REFERENCES}

Björklund R. A. and Magnussen S. (1981) A study of the interocular transfer of spatial adaptation. Perception 10, 511-518.

Blakemore C. and Campbell F. W. (1969) On the existence of neurones on the human visual system selectively sensitive to the orientation and size of retinal images. $J$. Physial., Lond. 203, 237-260.

Campbell F. W., Johnstone J. R. and Ross J. (1981) An explanation for the visibility of low frequency gratings. Vision Res. 21, 723-730.

Campbell F. W. and Robson J. G. (1968) Application of Fourier theory to the visibility of gratings $J$. Physiol., Lond. 197, 551-556.

Derrington A. M. and Badcock D. R. (1986) Detection of spatial beats: non-linearity or contrast increment detection? Vision Res. 26, 343-348.

De Valois K. K. (1977) Spatial frequency adaptation can enhance contrast sensitivity. Vision Res. 17, 1057-1065.

Georgeson M. A. and Harris M. G. (1984) Spatial selectivity of contrast adaptation: models and data. Vision Res. 24, 729-741.

Graham N. and Nachmias J. (197I) Detection of grating patterns containing two spatial frequencies: a comparison of single-channel and multi-channel models. Vision Res. 11, 251-259.

Jaschinski-Kruza W. and Cavonius C. R. (1984) A multiple-channel model for grating detection. Vision Res. 24, 933-941.

Klein S. and Stromeyer C. F. III (1980) On inhibition between spatial frequency channels: adaptation to complex gratings Vision Res. 20, 459-466.

Kelly D. H. and Burbeck C. A. (1980) Motion and vision III. Stabilized pattern adaptation J. opt. Soc. Am. 70, 749-754.

Magnussen S. and Greenlee M. W. (1986) Contrast threshold elevation following continuous and interrupted adaptation. Vision Res. 26, 673-675.

Nachmias J., Sansbury R., Vassilev A. and Weber A. (1973) Adaptation to square-wave gratings: in search of the elusive third harmonic. Vision Res. 13, 1335-1342.

Pantle A. and Sekuler R. (1968) Size-detecting mechanisms in human vision. Science, N.Y. 162, 1146-1148.

Quick R. F. (1974) A vector-magnitude model of contrast detection. Kybernetik 16, 1299-1302.

Sachs M. B., Nachmias J. and Robson J. G. (1971) Spatial frequency channels in human vision. J. opt. Soc. Am. 61, 1176-1186.

Sekuler R., Wilson H. and Owsley C. (1984) Structural modeling of spatial vision. Vision Res. 24, 689-700.

Stromeyer C. F. III and Klein S. (1974) Spatial frequency channels as asymmetric (cdge) mechanisms. Vision Res. $14,1409-1420$.

Tolhurst D. J. (1972) Adaptation to square-wave gratings: inhibition between spatial frequency channels in the human visual system. J. Physiol., Lond. 226, 231-248.

Tolhurst D. and Barfield L. P. (1978) Interactions between spatial frequency channels. Vision Res. 18, 951-958.

Watson A. B. and Robson 3. G. (1981) Discrimination at threshold: labelled detectors in human vision. Vision Res. 21, 1115-1122.

Wilson H. R. and Bergen J. R. (1979) A four-mechanism model for threshold spatial vision. Vision Res. 19. 19-32. Wilson H. R. and Gelb D. J. (1984) Modifed line element theory for spatial frequency and width discrimination. $J$. opt. Soc. Am. A1, 124-131.

\section{APPENDIX}

A quantitative estimate of the effect of adaptation at 3 rd and 9th harmonic frequencies on the sensitivity to low spatial frequency squarewave gratings was made using a revised version of the model presented by Jaschinski-Kruza and Cavonius (1984). Here, the contrast needed to detect a squarewave test grating is calculated based on the contrast sensitivity function before and after adaptation to a sinewave grating, the spatial frequency of which was either 1 or $3 \mathrm{c} / \mathrm{deg}$, or after sequential adaptation to these two gratings.

The model employs the following one-dimensional Gabor functions to map the sensitivity of symmetric receptive fields:

$$
\begin{aligned}
& w\left(x, x_{0}\right)=\exp \left[-\left\{\left(x-x_{0}\right) /\right.\right. \\
& \left.\left.\left(b^{*} T_{\varepsilon}\right)\right]^{2}\right]^{*} \sin \left[2 \pi^{*}\left(x-x_{0}\right) / T_{\mathrm{s}}\right]
\end{aligned}
$$

where $b$ determines the bandwidth and $T_{\mathrm{c}}$ is twice the width of the center lobe of the receptive field centered at $x_{0}$ : here $b=0.8$, giving a bandwidth at half-height of 1 octave. In the model, the center frequencies of the channels are separated by 0.17 logarithmic units (i.e. 6 channels per decade), which was constant over the entire spatial frequency scale. The spatial sampling interval is $0.01 \mathrm{deg}$, which corresponds to a density of 100 Gabor functions per degree. Probability summation was performed across channels and over space. This was conducted using the equation proposed by Quick (1974), with the exponent $\alpha=4.0$.

It is assumed that the contrast sensitivity to simple or complex gratings is determined by probability summation among the channels sensitive to the component frequencies. Adaptation at 1 and $3 \mathrm{c} / \mathrm{deg}$ reduces sensitivity of the channeis centered at, or near, those adapting frequencies, thus altering the shape of the contrast sensitivity function

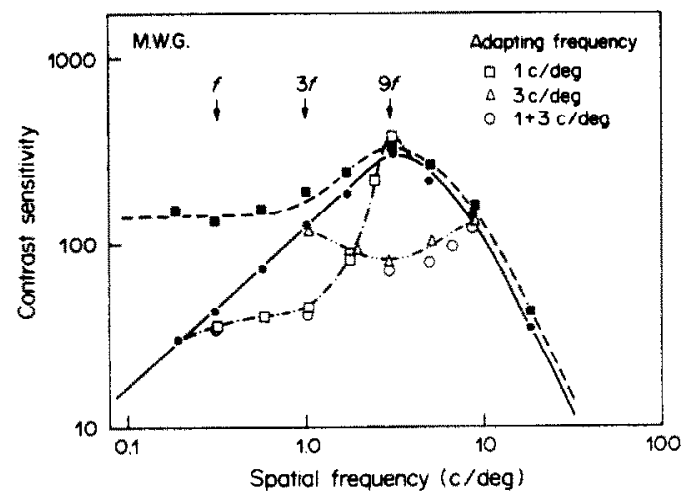

Fig. 6. Contrast sensitivity function (CSF) for sinewave (O) and squarewave gratings ( $\boldsymbol{D})$ before adaptation. Open squares show contrast sensitivity for sinewave gratings following adaptation to $1 \mathrm{c} / \mathrm{deg}$. Open triangles show contrast sensitivity for sinewaves following adaptation to $3 \mathrm{c} / \mathrm{deg}$, and open circles show contrast sensitivity following $8 \mathrm{~min}$ of sequential adaptation to 1 and $3 \mathrm{c} / \mathrm{deg}$. 


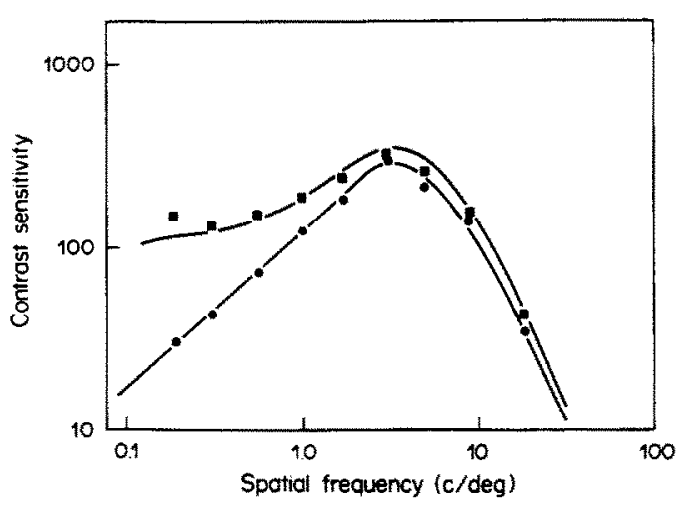

Fig. 7. Unadapted contrast sensitivity function for sinewave gratings (O) fitted by the polynominal used in the model to predict squarewave sensitivity $(\boldsymbol{\square})$ before harmonic adaptation. Symbols give observed values and continuous curves give model prediction.

(CSF) for sinewave gratings. Figure 6 shows the CSF for sinewave gratings before adaptation (O) and following $4 \mathrm{~min}$ adaptation at $1 \mathrm{c} / \mathrm{deg}(\square)$ and $3 \mathrm{c} / \mathrm{deg}(\Delta)$ and following $8 \mathrm{~min}$ of sequential adaptation at $\mathrm{l}$ and $3 \mathrm{c} / \mathrm{deg}$ (O). Here, it can be seen that the elevation in threshold caused by sequential adaptation at two spatial frequencies is approximately equal to the combined effects of continuous adaptation at each component frequency.

The sinewave CSFs found before and after harmonic adaptation were fitted by polynominals, which in turn were employed in the model to predict sensitivity to squarewave gratings. The results of these calculations for the unadapted sinewave CSF are shown in Fig. 7 by the continuous curve connecting the actual observed values (O). Predicted squarewave sensitivity is given by the curve connecting the observed sensitivity to squarewave gratings prior to adapta-

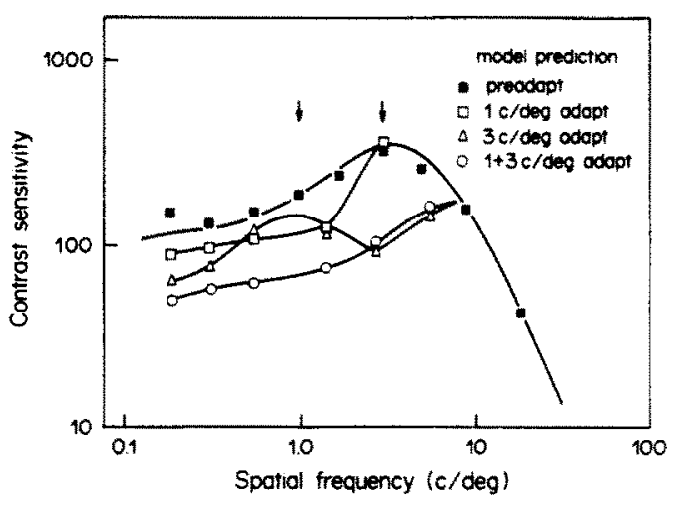

Fig. 8. Observed contrast sensitivity for squarewave gratings (D) before adaptation is compared to the predicted squarewave sensitivity following adaptation to a $1 \mathrm{c} / \mathrm{deg}(\square)$, $3 \mathrm{c} / \mathrm{deg}(\triangle)$ and to sequential adaptation to 1 and $3 \mathrm{c} / \mathrm{deg}$ $(O)$. Arrows denote position on the spatial frequency axis of the two adapting frequencies used in the experiments.

tion (C). Note that the model described above provides a good fit to the unadapted squarewave sensitivity.

Now let us consider the predictions made by the model based on the CSF measured after adaptation at harmonic frequencies of the $0.33 \mathrm{c} / \mathrm{deg}$ squarewave test grating. Figure 8 presents unadapted $(\square)$ values for squarewaves and the predicted values following $1 \mathrm{c} / \mathrm{deg}(\square), 3 \mathrm{c} / \mathrm{deg}(\Delta)$, and $1+3 \mathrm{c} / \mathrm{deg}(\mathrm{O})$ adaptation. Following sequential adaptation to 1 and $3 \mathrm{c} / \mathrm{deg}$, for example, the model predicted a $0.4 \mathrm{log}$ unit decrease in squarewave sensitivity at $0.33 \mathrm{c} / \mathrm{deg}$. However, we found no difference in squarewave sensitivity following this adaptation (see Fig. 4). Therefore, although the model holds for unadapted sensitivity to squarewaves, it cannot account for the persistence of this sensitivity following adaptation at the squarewave's harmonic frequencies. 